\title{
Facilitating Social Connectedness for People with Autism and Intellectual Disability Using an Interactive App
}

\author{
Mark Donnelly \\ Ulster University \\ Northern Ireland \\ mp.donnelly@ulster.ac.uk
}

\author{
Diane Hill \\ NOW Group \\ Northern Ireland \\ diane.hill@nowgroup.org
}

\author{
Raymond Bond \\ Ulster University \\ Northern Ireland \\ rb.bond@ulster.ac.uk \\ Pauline Fitzsimons \\ NOW Group \\ Northern Ireland \\ pauline.fitzsimons@nowgroup.org
}

\author{
Maurice Mulvenna \\ Ulster University \\ Northern Ireland \\ md.mulvenna@ulster.ac.uk
}

\author{
Suzanne Martin \\ Ulster University \\ Northern Ireland \\ s.martin@ulster.ac.uk
}

\author{
Laurence Taggart \\ Ulster University \\ Northern Ireland \\ l.taggart@ulster.ac.uk
}

\author{
Angela Hassiotis \\ University College London \\ England \\ a.hassiotis@ucl.ac.uk
}

\begin{abstract}
This short paper describes the evolution of a smartphone application, which aims to offer support for individuals living with an intellectual disability. The JAM (Just-a-minute) App enables people with a cognitive disability, such as those with an intellectual disability and Autistic Spectrum Disorder to inform service providers that they need 'Just A Minute' to get themselves organised, e.g. counting money. The app offers a simple and intuitive service that supports users to become more socially connected to the society around them by eliciting empathic responses from other actors in society. This paper describes the original non-digital version of the technology before presenting the current app version. The paper reports the success of the app through use case reports of its real-world use. It concludes with a reflection on the current app status and outlines the investigators' future plans to enrich the service with intelligent conversational interfaces (also known as chatbots).
\end{abstract}

Keywords: Mobile Design, Assistive Cognitive Technology, conversational interfaces, Intellectual disability, Autism Spectrum Disorder, Chatbot

\section{INTRODUCTION}

Globally, approximately 650 million people (10\%) are living with a disability, many of whom have a cognitive disability (UN Fact sheet on Persons with Disabilities). This includes those with an intellectual disability (ID) and Autistic Spectrum Disorder (ASD). Unsurprising, such figures place ID and among the top 10 most expensive disabilities, in Europe. People living with these disabilities may exhibit problems with attention and memory; difficulties in processing information, perception, reasoning, problem-solving, self-monitoring and selfawareness; comprehension, communication and problems with adaptive functioning. Consequently, they face challenges when attempting to undertake typical everyday tasks, such as managing personal finances, maintaining personal care, accessing public transport, shopping, food preparation, selfdirected healthcare and communication. These tasks are made more challenging as services and technologies do not always specifically cater for the individual needs of users with ID and ASD. In addition, there is often a lack of empathy and knowledge amongst service providers regarding the needs of users with disabilities. Consequently, there is a potential for the social connectedness of these individuals to be strained, creating barriers towards engaging in everyday society.

Assistive technology has been reported to offer support towards increasing the quality of life and independence of people living with disabilities, however, research has argued that while people with ID or ASD potentially stand to gain the most from recent advancements in technology, they are traditionally the group within society least likely to gain access to and receive the full benefits from such developments' [Chadwick et al., 2013]. Such reports illustrate a need for further research into assistive technologies and cognitive-disability-aware services that meet the needs of people with ID and ASD. This paper presents the development of a simple, yet novel assistive technology that aims to ease communication barriers for people living with ID and ASD, and to encourage empathy among service providers.

The sections of the paper are organised as follows: Section 2 highlights exemplar research into assistive technologies, developed for people with ID or ASD; 
Sections 3 describes the development and status of the JAM App; Section 4 presents a number of real user cases. Finally, Section 5 explores opportunities and concludes this paper with an outline plan of our future work, to embed intelligent conversation interfaces into the technology to enhance current services.

\section{RELATED WORK}

Assistive technology can be defined as "any item, piece of equipment, or product system, whether acquired commercially off the shelf, modified, or customized, that is used to increase, maintain, or improve the functional capabilities of a person with a disability" (Bjørneby, Topo, \& Holthe, 1999). Whilst this traditionally has involved physical solutions, there has been recent attention made to assistive technologies for cognition (ATC) also known as cognitive assistants.

Recent appraisals of ATCs [de la Guia et al., 2015; Shpigelman, 2016; Caton \& Chapman, 2016) highlight that most have been developed for the general public in the form of calendars, reminder services and to-do lists. Consequently, there is a need to tailor ATCs to help people with cognitive disabilities. ATCs for people with ID or ASD have mainly surrounded 'sequencing' devices. These are devices that help users by guiding them through tasks such as cooking step-by-step. Sequencing devices often include voice activated commands or visual prompts, but these are limited to unidirectional communication. What follows is a brief overview of some of existing ATCs:

- Planning and Execution Assistant (PEAT): one of the first sequencing systems which incorporated elementary artificial intelligence and Voice Mediated ATC.

- Neuropage: one of the first reminding technologies for people with cognitive disabilities and ID. The reminders were used for medication prompting, social events and everyday activities.

- Pocket-Coach: a dedicated 'sequencing device' for people with cognitive disabilities. It allows carers/family members to deconstruct 'everyday' tasks into steps. The sequencing device then uses these steps with audio prompts to guide the user through completing an everyday task.

- LifePal: an app supporting young people with ASD by helping them organize their routine, complete daily tasks, receive reminder prompts, encourage routine reflection and to provide travel assistance. Four core personalised services are delivered via a smartphone App: task management combined with gamification (positive reinforcement via a reward system) to recognize achievements and encourage and promote engagement; life-logging of emotions and feelings to promote improved understanding of behaviours, thoughts and moods; travel support via geo-fencing and priority calling services to promote independent travel while safeguarding users' wellbeing; and structured reminding support [ref].

- Look at Me: an app from Samsung which attempts to support users with ID and ASD to read and interpret the visual expressions of those with whom people they come into contact.

A key element for a successful ATC is what is referred to as zero effort technology. According to Sayko et al. (2015) in their most recent review of ATCs, the "interface continues to be a major design issue for all these systems" and ATCs have not realised their full potential because they require intricate interactions that are difficult for people with ID or ASD with a range of cognitive impairments and communication difficulties.

\section{JAM CARD APP DEVELOPMENT}

NOW Group is a Northern Ireland based social enterprise who support people with ID and ASD into employment, training, transition and volunteering. In 2012, NOW launched the innovative JAM ('Just a Minute') Card that enables people with an ID and ASD to tell others that they need Just a Minute. The JAM Card (Figure 1) provides valuable, non-verbal communication for a person with a cognitive disability; that can also include those living with a acquired brain-injury, dementia, stroke, etc.

While people of all ages experience difficulties with their disability, the needs of younger people are paramount as they seek to understand and navigate social norms in mainstream education and their communities.

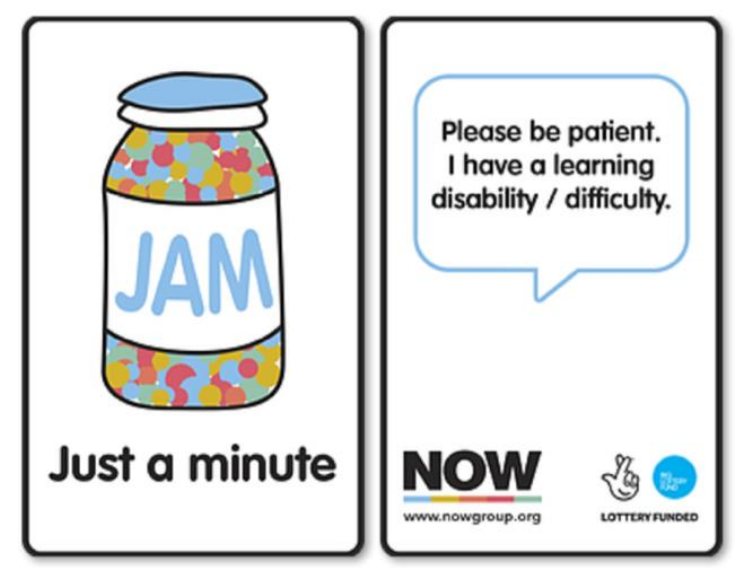

Figure 1: The JAM Card.

The JAM Card was the idea of NOW Service Users who said that they often felt under pressure in public and when using services, and would like a discreet way of letting service providers know that they need a little extra time and patience while, for example, making shop purchases or accessing public 
transport. The JAM Card is available as a credit card sized card that can fit in a wallet.

The JAM Card has been and continues to a great success with over 8,000 JAM Cards in use across Northern Ireland, with many requests for these cards from the UK, Ireland and further afield across Europe. Based on the 2011 Census data, this uptake equates to around $20 \%$ of the 40,000 Northern Ireland population living with a communication, learning, intellectual or social difficulty. JAM Card can be used by people with ID and ASD but has also been used by those who have acquired brain injuries, dementia, stroke and people who otherwise feel self-conscious about their ability to communicate effectively when engaging with others.

Continued engagement with NOW Service users, led by NOW, highlighted an optimism and desire among users to evolve JAM Card into a digital assistive technology through the use of smart phones. Launched in January 2017, the JAM Card App (Figure 2) offers the same core functionality as the above-mentioned JAM Card but additionally, allows the user to select with which service the user used the card. The app then guides the user to rate their experience of that particular service.

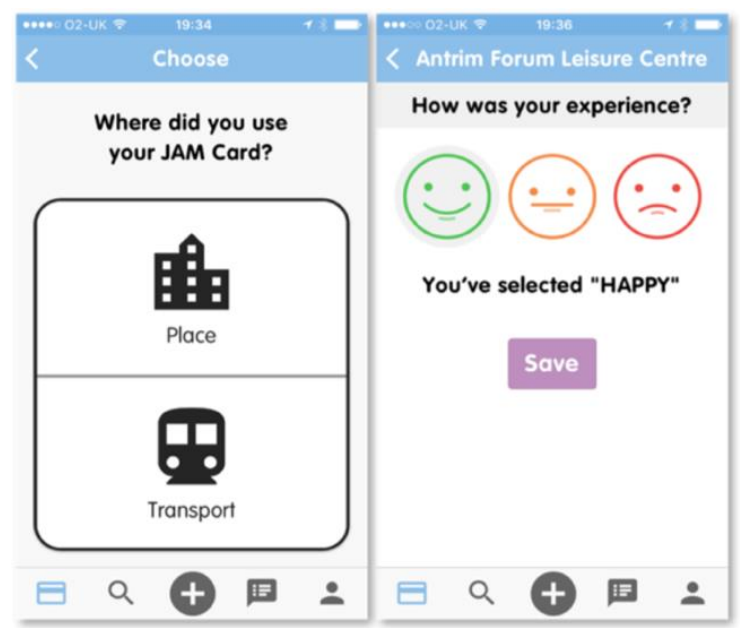

Figure 2: JAM Card App, available on Android \& iOS

The App echoes the original JAM Card goals, inducing empathy in service providers and providing essential everyday support to end-users through simple, intuitive and guided design. The principle human-computer interface factor influencing the design of the JAM Card App is simplicity of use. Background Application Programming Interface (API) services track and prompt users to confirm their geolocation upon which a wizard-based approach, guides users through a series of screens, to complete and log each app usage.

The JAM Card App was released globally via the Apple App and Google Play stores as a free app, in January 2017. To date, there have been more than 1243 downloads (figures correct as of May 2018). Users of the JAM Card App have engaged enthusiastically, providing valuable data, including usage geographically (Figure 3 ) and by Service Provider (Figure 4), showing a wide variety of Service Provider engagements: Travel, Eating Out, Shopping, Learning, Services and Entertainment.

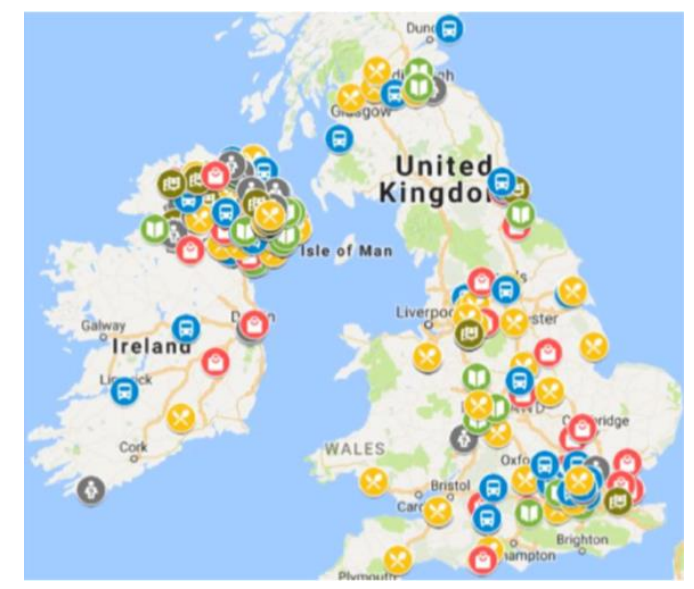

Figure 3: JAM Card App Usage within UK \& Ireland

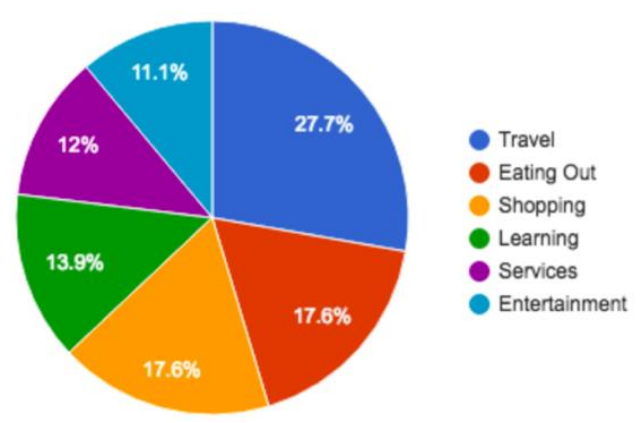

Figure 4: JAM Card App Usage by Service Provider

\section{USE CASE REPORTS}

Evidence of the early success of JAM Card App is presented in this section A series of short use case reports serve to illustrate the range of settings and diversity of users engaging with the technology.

\subsection{User Reports}

A young woman with a cognitive disability used her JAM Card in a busy fast food restaurant. She felt rushed by the person at the cashier's desk as she tried to get her money counted. Once she showed the JAM Card, the staff member told her to take as much time as she needed. She said it made her feel happy and relaxed and more confident about going out on her own and trying new places.

A woman in her 40's with an ID used her JAM Card App with a bus driver when travelling into town. She reported that the driver was being impatient with her as she counted out her fare. She showed him the JAM Card App message of 'Just a minute' and 'Please be patient. I have a learning disability'. He immediately stopped rushing her and gave her 
plenty of time. The woman reported that the card made her feel liberated and helps her in daily life.

A woman in her early 20's with an ID has been using the JAM Card almost everyday since she downloaded it. She mostly uses it on public transport when people try to push past her or if the driver is hurrying her along while she sorts out her money. She says that when she shows people the JAM Card they understand what she is requesting and become more patient. She likes using the JAM Card because of the understanding it gives people of her situation.

A young woman with ID fell badly in Belfast. She cut her face and knocked out a number of her teeth. She made her way to the general hospital and was extremely distressed and unable to communicate to the reception staff. She showed the receptionist the JAM Card and the receptionist automatically left her desk and came straight to her to offer extra help and patience. The young lady reported that the extra time offered to her helped to ease her stress.

\subsection{Care-giver Reports}

To complement these use cases, we reflect upon some of the feedback gleaned from care-givers and professional practitioners.

The mother of one JAM Card user reported that her daughter uses it when she is shopping, at the cinema and in restaurants. Her confidence has increased so much that she is now planning a weekend in Dublin with her friends and older sister, something she would not have done before.

A speech and language therapist who assists vulnerable adults in court has downloaded the app and hopes to introduce it to some of the people she assists while giving evidence at court who need extra time to process questions.

The parent of a JAM Card user downloaded the app to use with her son who has ASD, has severe ID and challenging behaviour. He likes to go into the same shops with his carers over and over again. The shop staff became suspicious of his behaviour and the JAM Card has helped to explain his situation.

\section{DISCUSSION AND CONCLUDING REMARKS}

This paper presents an overview of JAM Card and the developments of the JAM Card App. While the current JAM Card App has been successfully used for simple non-verbal messaging to facilitate conversations between a service provider and users with ID and ASD, there is scope to investigate the opportunity to enrich the JAM Card App further. Our roadmap for the continued development of the JAM Card App will involve the integration of intelligent conversational interfaces. Specifically, the app will facilitate research into the delivery of frictionless interfaces for enhanced cognitive engagement with users. Such a chatbot (or 'JAMBot') may facilitate natural human-computer interactions that can provide personalised context-aware and affectiveaware assistance and guidance to users whilst they undertake everyday tasks (online and in the real world). JAMBot will assist users during specific everyday tasks and augment user's reasoning and executive function whilst building the user's selfadvocacy and confidence to interact and engage with offline and online services of which they would ordinarily not have taken advantage. Chatbot technology can also signpost and provide intelligent communications based on a personalised profile as well as using an ontology of contextual and emotional intelligence. It can involve a digital communication mediation card builder which uses tailored messages on a smart-phone screen to mediate communication experiences between the user and everyday service providers.

Utilising these technologies will serve to further improve the social connectedness of people living with ID and ASD as they engage with and consume local services.

\section{REFERENCES}

Bjørneby, S., Topo, P., \& Holthe, T. (1999). Technology, ethics and dementia: A guide- book on how to apply technology in dementia care. Oslo, Norway: The Norwegian Centre for Dementia Research, INFO-Bank. Norwegian Centre for Dementia Research, INFO-banken.

Chadwick D. Fullword C. \& Wesson C. (2013): Intellectual Disability, Identify and the Internet. In Handbook of Research on Technoself: Identity in a Technological Society; Luppicinil R., Ed.; IGI Global: Hershey, PA, USA, 2013; pp. 229-254.

Caton S, Chapman M. The use of social media and people with intellectual disability; a systematic review and thematic analysis. Journal of Intellectual and Developmental Disability. 2016, 2; 41(2):125-39.

De la Guia E, Lozano MD, Peniche VM. Tangible User Interfaces to Digitise Real Environment. IEEE Transactions. 2015; 13(12); 3997-4003.

Sayko ME, Tremoulet P. Assistive Technologies for People with Cognitive Disabilities: Challenges and possibilities. In 2015 AAAl Fall Symposium, 2015 Sep 23.

Shpigelman CN. Leveraging Social Capital of Individuals with Intellectual Disability through Participation on Facebook. Journal of Applied Research in Intellectual Disabilities. 2016 Dec 1.

UN Fact sheet on Persons with Disabilities, http://www.un.org/disabilities/documents/ toolaction/pwdfs.pdf; last accessed 04/05/18. 